\title{
Evolutionary Analysis of MBW Function by Phenotypic Rescue in Arabidopsis thaliana
}

\author{
Bipei Zhang and Martin Hülskamp* \\ Botanical Institute, Biocenter, University of Cologne, Cologne, Germany
}

\section{OPEN ACCESS}

Edited by:

Stefan A. Rensing,

University of Marburg, Germany

Reviewed by:

Rainer Melzer

University College Dublin, Ireland

Catalin Voiniciuc,

Heinrich Heine University Düsseldorf,

Germany

*Correspondence:

Martin Hülskamp

martin.huelskamp@uni-koeln.de

Specialty section:

This article was submitted to Plant Development and EvoDevo,

a section of the journal

Frontiers in Plant Science

Received: 04 January 2019

Accepted: 12 March 2019

Published: 29 March 2019

Citation:

Zhang B and Hülskamp M (2019) Evolutionary Analysis of MBW

Function by Phenotypic Rescue

in Arabidopsis thaliana.

Front. Plant Sci. 10:375.

doi: 10.3389/fp/s.2019.00375
The MBW complex consisting of the three proteins R2R3MYB, $\underline{\mathrm{b}} H L H$ and $\underline{W} D R$ regulates five traits in Arabidopsis thaliana including trichome and root hair patterning, seed coat color, anthocyanidin production and seed coat mucilage release. The WDR gene TTG1 regulates each trait in specific combinations with different bHLH and R2R3MYB proteins. In this study we analyze to what extent the biochemical properties of the MBW proteins contribute to trait specificity by expressing them in appropriate A. thaliana mutants. We show that the rescue behavior of $A$. thaliana bHLH and R2R3MYB protein is sufficient to explain the function as derived previously from mutant analysis. When extending this rescue approach using MBW proteins from other species we find that proteins involved in anthocyanidin regulation typically show a rescue of the anthocyanidin phenotype but not of the other traits. Finally, we correlate the rescue abilities of MBW protein from different species with the $A$. thaliana proteins.

\footnotetext{
Keywords: MBW complex, protein function, Arabidopsis thaliana, Arabis alpina, Gossypium hirsutum, Petunia hybrida, Zea mays
}

\section{INTRODUCTION}

In the model plant Arabidopsis thaliana five traits including trichome and root hair patterning, seed coat color, anthocyanidin production and seed coat mucilage release are genetically linked through the action of the TRANSPARENT TESTA GLABRA1 (TTG1) gene (Koornneef, 1981). Mutations in the TTG1 gene strongly affect all five traits. TTG1 encodes a WDR protein (Walker et al., 1999) that forms protein complexes with R2R3MYB and basic Helix-Loop-Helix factors ( $\underline{b} H L H)$ that transcriptionally regulate downstream target genes (for review see Lloyd et al., 2017). This complex is called the MBW complex (Ramsay and Glover, 2005; Xu et al., 2015; Zhang and Schrader, 2017) and it forms through the binding of TTG1 and the MYB protein to the bHLH protein (Payne et al., 2000; Zhang et al., 2003; Zimmermann et al., 2004; Feller et al., 2006). In addition, most bHLH proteins dimerise suggesting that higher ordered protein complexes are possible (Payne et al., 2000; Bernhardt et al., 2003; Zhang et al., 2003). While TTG1 is a single copy gene, there are four bHLH genes including GL3 (Hülskamp et al., 1994; Payne et al., 2000; Bernhardt et al., 2003; Zhang et al., 2003; Gonzalez et al., 2008; Feyissa et al., 2009), EGL3 (Bernhardt et al., 2003; Zhang et al., 2003; Gonzalez et al., 2008), MYC1 (Symonds et al., 2011; Bruex et al., 2012) and TT8 (Nesi et al., 2000; Zhang et al., 2003; Baudry et al., 2006) involved. The genetic analysis revealed some trait specificity such that each bHLH is genetically important for two or more of the five traits. The R2R3MYB genes involved in the regulation of the five TTG1-dependent traits are represented by several genes. These display high trait specificity. Trichome patterning is regulated 
by GL1 and MYB23 (Herman and Marks, 1989; Marks and Feldmann, 1989; Oppenheimer et al., 1991; Kirik et al., 2005), root hair patterning by WER (Lee and Schiefelbein, 1999), seed coat mucilage by MYB61 (Penfield et al., 2001; Zhang et al., 2003) and MYB5 (Gonzalez et al., 2009), seed color (proanthocyanidin production) by TT2 (Baudry et al., 2004; Gonzalez et al., 2009) and anthocyanidin by PAP1 and PAP2 (Borevitz et al., 2000; Teng et al., 2005; Gonzalez et al., 2008). Taken together these findings led to the concept that different MBW complexes discriminate between different traits. This raises the question, whether this specificity is due to different protein functions or different transcriptional regulation. On the one hand, target gene specificity has been mapped to a few relevant amino acids in the MYB proteins TT2 and PAP4 highlighting that specificity is mediated at the level of protein functions (Heppel et al., 2013; Lai et al., 2013). On the other hand, there is some evidence available for R2R3MYB proteins suggesting that the proteins have equivalent functions and that differences in the transcriptional regulation lead to the observed genetic specificity. GL1 and WER are excellent examples as GL1 expressed under the WER promoter can rescue the wer root hair phenotype and vice versa (Lee and Schiefelbein, 2001). The bHLH proteins may have properties that are relevant for the specific traits. This is exemplified by the ability of GL3 and EGL3 to rescue myc1 mutants under the MYC1 promoter whereas MYC1 cannot rescue $g l 3$ egl3 mutants under the GL3 or the EGL3 promoters (Zhao et al., 2012). Thus, there is evidence available for both possibilities, specificity by transcriptional regulation and protein specificity (Lai et al., 2013; Lloyd et al., 2017).

The MBW network is evolutionary conserved in plants and was reported in monocots, dicots, angiosperms and gymnosperms (Ramsay and Glover, 2005; Quattrocchio et al., 2006a; Serna and Martin, 2006; Xu et al., 2015; Nemesio-Gorriz et al., 2017; Zhang and Schrader, 2017). The regulation of the flavonoid pathway is considered to be the most ancient role of this complex. It is postulated that additional functions have evolved by gene duplications followed by functional diversification after rosid-asterid split (Serna and Martin, 2006). Consistent with this, mutations in the WDR gene TTG1 in Arabis alpina and Matthiola incana lead to phenotypes in all five traits affected in A. thaliana (Koornneef, 1981; Dressel and Hemleben, 2009; Chopra et al., 2014). Functional conservation between Arabidopsis and more distantly related species were frequently assessed by rescuing the respective Arabidopsis mutants by overexpressing genes under ubiquitous promoters or gene-specific promoters (see references in Supplementary Table S1 for details).

In this study, we aimed to understand to what extent MBW protein function is relevant for rescuing each of the five TTG1dependent traits in Arabidopsis mutants. Toward this end, we expressed the MBW genes under the 35S promoter in the respective mutants and compare our results to previously published results. In a first step, we studied Arabidopsis genes. For bHLH genes we show that their functional specialization can be explained by specialized protein function. In a second step, we analyzed MBW proteins from other species including Arabis alpina, cotton (Gossypium hirsutum), petunia (Petunia x hybrida) and maize (Zea mays). Finally, we assessed the ability of MBW proteins from other species to interact with Arabidopsis MBW proteins to judge the relevance of changes in the protein-protein interactions for rescue ability.

\section{MATERIALS AND METHODS}

\section{Arabidopsis Strains and Plant Growth}

The mutant alleles used in this study: ttg1-1 (Ler) (Koornneef, 1981; Walker et al., 1999). gl3-3 (Col) background (Jakoby et al., 2008); egl3-77439 (Col) background (TAIR accession 1008704039); tt8-048673 ( Col) background (TAIR accession 1005848854); gl3 egl3 (Col) (Friede et al., 2017); gl3/egl3/tt8 (homozygous progeny by crossing gl3/egl3 with tt8-048673); gl1 (Col) (Oppenheimer et al., 1991); pap1 (pst16228) (No-0; CS28564) (Kuromori et al., 2004); pap2 (Col) (salk_093731, TAIR accession 1005457343). Plants were grown on soil at $24^{\circ} \mathrm{C}$ with $16 \mathrm{~h}$ of light per day. All transgenic plant lines used and created in this work are listed in Supplementary Table S2. Transgenic lines were generated using the floral dip method (Clough and Bent, 1998). Transgenic T1 plants were selected on $1 / 2$ MS agar plates containing $10 \mathrm{mg} / \mathrm{L}$ BASTA [glufosinate ammonium, SigmaAldrich (Munic, Steinheim)].

\section{Constructs}

All CDS entry clones were generated by amplifying the CDSs from start to stop codon from A. thaliana (At), Arabis alpina $(\mathrm{Aa}), \mathrm{G}$. hirsutum (Gh), Petunia hybrid $(\mathrm{Ph})$, and Zea mays $(\mathrm{Zm})$ followed by BP recombination in pDONR201/207.

\section{LUMIER Vectors}

Three different destination vectors were used for subsequent LR reactions. pcDNA3-Rluc-GW and pTREX-dest30 (Invitrogen) for the $\mathrm{N}$-terminal fusion of Renilla reniformis and Staphylococcus aureus protein, respectively, were described before (Pesch et al., 2015).

Genes were N-terminal fused to the $S$. aureus protein A sequence in pTREX-dest30-ntProtA by LR reaction. For the negative control, the vector pTREX-dest30-ntProtA was recombined with pENTR1A-w/occdB.

The full-length Renilla luciferase sequence was N-terminal fused to the coding sequences in pcDNA3-Rluc-GW. Also pENTR1A-w/o-ccdB was recombined to this vector as a negative control.

\section{Plant Vectors}

All CDS of MBW homologs were cloned into Donor vectors by $\mathrm{BP}$ reactions (Invitrogen). Then recombination of the corresponding entry clones with the $35 \mathrm{~S}$ promoter containing vector pAMPAT-35S-GW [GenBank accession no. AY436765 (Pesch et al., 2015)].

\section{Phenotypic Analysis}

The presence of trichomes was scored on leaves 1-6 on 2-weekold seedlings. For the analysis of root hairs $\mathrm{H}$-files were identified microscopically by their position over cortical cell boundaries 
in roots of 7-day-old 1/2MS plate-grown T2 progeny seedlings The number root hairs in the flanking $\mathrm{N}$-files was roughly determined in 1-week-old basta-resistant T2 seedlings under a Leica stereomicroscope (MZ FLIII).

Anthocyanidin was analyzed in T2 seedlings grown on 1/2MS germination medium containing 3\% sucrose, and analyzed using a Leica stereomicroscope (MZ FLIII) with the Multi-Focus and Montage option of the Leica Application Suite V3 (Leica Microsystems, Wetzlar, Germany). Seed coat color was observed in T1 progeny seeds and pictures were taken with a Canon EOS 5D Mark (Canon, Krefeld, Germany). For the analysis of seed mucilage T2 seeds were imbibed in $0.2 \% \mathrm{w} / \mathrm{v}$ aqueous ruthenium red (Sigma) solution with $0.5 \%$ agar and monitored after $3-4 \mathrm{~h}$ by light microscopy and pictures taken using the DISKUS software (Carl H. Hilgers Technisches Büro, Germany) (Modified from Penfield et al., 2001).

\section{LUMIER (LUminescence-Based Mammalian IntERactome)}

For LUMIER assays, each protein was transiently expressed in HEK293TM cells (BioCat/SBI: LV900A-1) as hybrid proteins either with the $S$. aureus protein A (ProtA) or with the $R$. reniformis luciferase (Rluc) fused to their amino $\mathrm{N}$ termini. The LUMIER assay is based on the technique established for high throughput screens (Blasche and Koegl, 2013) and was optimized for the proteins used in this study (Pesch et al., 2013; Pesch et al., 2015). Constructs (1.5 $\mu \mathrm{g}$ plasmid DNA) were singletransfected into $1 \times 10^{6} \mathrm{HEK} 293 \mathrm{TM}$ cells in 6-well plates using $10 \mu \mathrm{l}$ Lipofectamine 2000 (Thermo). After $48 \mathrm{~h}$, the medium was removed and cells were washed with PBS. The cells were collected by centrifugation (600 g, $15 \mathrm{~min}$ ) and lysed on ice in $50 \mu \mathrm{l}$ icecold lysis buffer $[20 \mathrm{mM}$ Tris pH 7.5, $250 \mathrm{mM} \mathrm{NaCl}, 1.1 \%$ Triton X-100, 10 mM EDTA, 10 mM DTT, Protease Inhibitor Cocktail (Roche, 1836170) and benzonase (Novagen, 70746; 0.0125 units per $\mu \mathrm{l}$ final concentration)] for $1 \mathrm{~h}$. Subsequently, lysates were centrifuged at $15,000 \mathrm{rpm}(21,380 \mathrm{~g})$ for $15 \mathrm{~min}$ at $4^{\circ} \mathrm{C}$, and the supernatant was mixed with $10 \mu \mathrm{l}$ PBS-prewashed sheep anti-rabbit IgG-coated magnetic beads (Invitrogen, Dynabeads $\mathrm{M} 280 ; 2 \mathrm{mg} / \mathrm{ml}$ final concentration) and incubated for $1 \mathrm{~h}$ on ice. The beads were collected using a magnetic holder (Neodym-Magnets, 1.3 Teslar) and luminescence measured in a microtiter plate reader (BMG Fluostar Optima). The pulldown was also performed with untransfected cells and with cells solely expressing Luciferase-protein to exclude any non-specific signal from the cell lysate and non-specific binding of Luciferaseprotein to the beads, respectively.

The percentage of Rluc on the beads compared with the lysate was calculated by dividing the Rluc activity on the beads by the Rluc activity in the same amount of lysate used in the pulldown assay (Input). Pulldown efficiency was calculated by: [Rluc pulldown/Input] $\times 100$.

\section{Phylogenetic Tree}

Unrooted Maximum Likelihood Phylogenetic Trees for WDR, bHLH, and R2R3MYB was done with MEGA6 (Tamura et al., 2013) using entire amino acid sequences based on the JTT matrix-based model (Jones et al., 1992). In addition to the proteins used in this study we added proteins from other species also discussed to play a role in anthocyanidin regulation (Zhang and Schrader, 2017).

\section{RESULTS}

In order to study the functional conservation of MBW proteins we performed rescue experiments in suitable $A$. thaliana mutants under the $35 \mathrm{~S}$ promoter. We used the same strong promoter for all experiments to facilitate a direct comparison of the protein properties in all experiments. Although this strategy has the disadvantage that our results may differ to published experiments using gene-specific promoters (Supplementary Table S1) we consider this to be a reasonable procedure to compare the protein properties. For completeness and for comparison in our assay system we also included genes for which this type of rescue data were already published.

Rescue of all five traits were analyzed qualitatively in the T1 generation (Figure 1) to judge the rescue efficiency at different chromosomal insertions. Trichomes were scored on rosette leaves of 3-week-old seedlings. The pro-anthocyanidin and seed coat mucilage phenotype were assayed in T2 seeds because both are maternal traits. Also anthocyanidin production and root hairs were studied in the T2 generation for technical reasons. However, rescue was still compared at the level of the $\mathrm{T} 1$ generation as represented by analyzed T2 plants. We studied anthocyanidin production in the hypocotyl on 4 days old seedlings grown on MS with 3\% sugar by visual inspection (Teng et al., 2005). Seed coat mucilage was analyzed by staining the mucilage with Ruthenium Red (Penfield et al., 2001). Pro-anthocyanidin production was inspected by seed color and root hairs by assessing trichomes in $\mathrm{N}$-file files of 1-week-old basta-resistant T2 seedlings.

For our functional comparison we initially tested the Arabidopsis MBW proteins for their ability to rescue the different traits. Then we selected MBW proteins from other species including A. alpina, cotton (Gossypium hirsutum), petunia (Petunia $x$ hybrida) and maize (Z. mays). Species and genes were chosen based on already published information suggesting that the MBW genes are relevant in the regulation of the traits considered here. The phylogenetic relationship of the proteins is shown in Supplementary Figure S1.

\section{Rescue of the Arabidopsis ttg1 Mutant by AtTTG1 Homologs}

In order to analyze whether the protein function of homologous TTG1 genes in other species is conserved we performed rescue experiments in A. thaliana ttg1-1 mutants (Koornneef, 1981; Walker et al., 1999). Toward this end we selected TTG1 homologs from several species including A. thaliana (AtTTG1), A. alpina (AaTTG1), G. hirsutum (GhTTG1, GhTTG2, GhTTG3, GhTTG4), P. hybrida (PhAN11) and Z. mays (ZmPAC1, ZmMP1). A. thaliana TTG1 served as a control for the rescue efficiency.

Arabidopsis thaliana and A. alpina TTG1 showed efficient rescue of all traits in most T1 lines (Table 1). The cotton GhTTG1 and GhTTG3 proteins also rescued all traits in more than $50 \%$ 

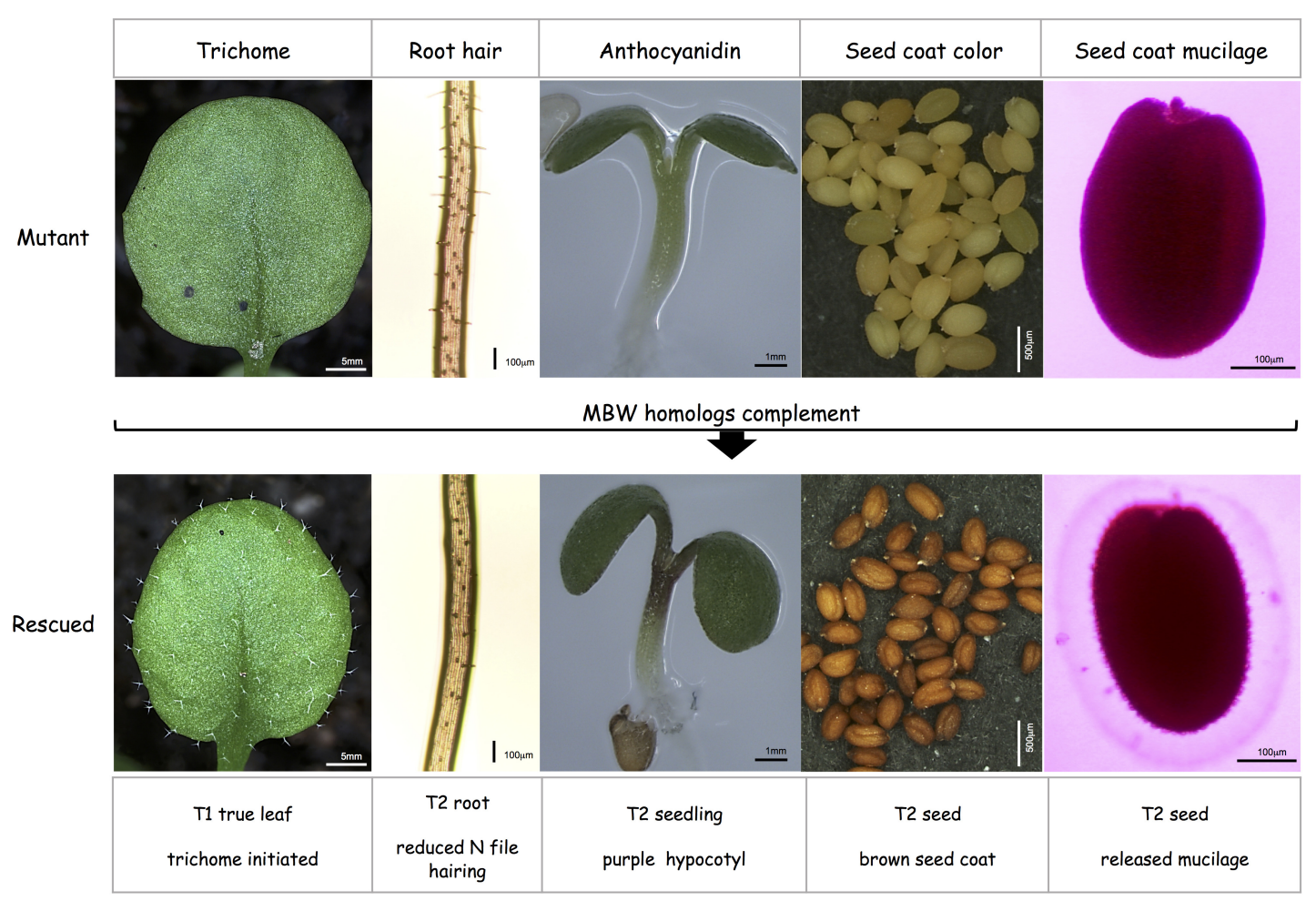

FIGURE 1 | Rescue strategy for the five TTG1-dependent traits in Arabidopsis mutants. (Top row) ttg1 mutant or g/3 eg/3 tt8 triple mutant phenotypes display glabrous rosette leaves, extra root hairs in $\mathrm{N}$-files, no anthocyanidin production under stress conditions in the hypocotyl, yellow seeds and no seed coat mucilage release. (Bottom row) rescue by the expression of MBW homologs: recovery of trichomes, suppression of extra root hairs in $\mathrm{N}$-files, purple hypocotyls under stress conditions, brown seeds and the release of seed coat mucilage (light pink Ruthenium Red labeled region around the seed).

TABLE 1 | T1 plants or T2 lines transformed with PAMPAT-35S-WDR showing phenotypic rescue (rescued lines/total number of lines) in ttg1.

\begin{tabular}{|c|c|c|c|c|c|}
\hline Gene & Trichome & Root hair & Anthocyanin & $\begin{array}{l}\text { Seed coat } \\
\text { color }\end{array}$ & $\begin{array}{l}\text { Seed coat } \\
\text { mucilage }\end{array}$ \\
\hline AtTTG1 & $25 / 27$ & $23 / 27$ & $22 / 27$ & $25 / 27$ & $25 / 27$ \\
\hline AaTTG1 & $15 / 16$ & $13 / 16$ & $12 / 16$ & $13 / 16$ & $14 / 16$ \\
\hline GhTTG1 & $35 / 50$ & $33 / 50$ & $33 / 50$ & $35 / 50$ & $35 / 50$ \\
\hline GhTTG2 & $0 / 15$ & $0 / 15$ & $0 / 15$ & $0 / 15$ & $0 / 15$ \\
\hline GhTTG3 & $14 / 19$ & $12 / 19$ & $12 / 19$ & $13 / 19$ & $13 / 19$ \\
\hline GhTTG4 & $0 / 16$ & $0 / 16$ & $0 / 16$ & $0 / 16$ & $0 / 16$ \\
\hline PhAN11 & $12 / 15$ & $12 / 15$ & $12 / 15$ & $12 / 15$ & $12 / 15$ \\
\hline ZmPAC1 & $34 / 35$ & $30 / 35$ & $30 / 35$ & $32 / 35$ & $34 / 35$ \\
\hline$Z m M P 1$ & $0 / 12$ & $0 / 12$ & $0 / 12$ & $0 / 12$ & $0 / 12$ \\
\hline
\end{tabular}

Bold values indicate rescue.

of the T1 lines [see also (Humphries et al., 2005)]. GhTTG2 and GhTTG4 did not show rescue which is consistent with the previous result that transient expression cannot rescue the anthocyanidin phenotype of the ttg1 mutation in M. incana (Humphries et al., 2005). Petunia AN11 efficiently rescued all traits in most T1 lines [see also (Payne et al., 2000)]. Consistent with previous results, the maize PAC1 also rescued most T1 lines (Carey et al., 2004). MP1 expression did not rescue the ttg1 phenotypes.

\section{Rescue of the Arabidopsis g/3 eg/3 tt8 Mutant by bHLH Homologs}

bHLHs proteins involved in MBW functions in the context of the five traits analyzed are typically important for some but not all traits. In order to study the function of bHLH homologs in Arabidopsis we used the gl3 egl3 tt8 triple mutant in Col background. This triple mutant exhibits defects in all five traits similarly as ttg1 mutants (Zhang et al., 2003).

In a first step, we studied the rescue ability of the Arabidopsis bHLH proteins AtGL3, AtEGL3, and AtTT8 (Table 2). The genetic analysis had revealed partially overlapping functions of these genes such that trichome and root hair formation are controlled by AtGL3, AtEGL3, anthocyanidin production by AtGL3, AtEGL3, and AtTT8, seed color by AtTT8, seed coat mucilage by AtEGL3 and AtTT8 (Zhang et al., 2003). To distinguish, whether this differential function is due to differential expression or different protein functions, we expressed the respective CDS in the $g l 3$ egl3 tt8 mutant. In addition, we included AtMYC1 which is involved in trichome and root hair development but has a molecular function distinct from AtGL3 and AtEGL3 and cannot rescue the gl3 egl3 mutant trichome phenotype (Zhao et al., 2012; Pesch et al., 2013). We noted that none of the genes rescued seed coat color. We reasoned that the $35 \mathrm{~S}$ promoter might not be sufficient for driving the expression in the seed coat. We therefore performed a second 
TABLE 2 | T1 plants or T2 lines transformed by pAMPAT-35S:bHLH showing phenotypic rescue (rescued lines/total number of lines) in g/3/eg/3/tt8.

\begin{tabular}{lccccc}
\hline Gene & Trichome Root hair Anthocyanin & $\begin{array}{c}\text { Seed coat } \\
\text { color }\end{array}$ & $\begin{array}{c}\text { Seed coat } \\
\text { mucilage }\end{array}$ \\
\hline AtGL3 & $\mathbf{1 8 / 2 0}$ & $\mathbf{1 4 / 2 0}$ & $\mathbf{1 8 / 2 0}$ & $0 / 20$ & $0 / 20$ \\
AtEGL3 & $\mathbf{1 5 / 2 0}$ & $\mathbf{1 0 / 2 0}$ & $\mathbf{1 0 / 2 0}$ & $0 / 20$ & $\mathbf{9 / 2 0}$ \\
AtMYC1 & $0 / 20$ & $\mathbf{7 / 2 0}$ & $0 / 20$ & $0 / 20$ & $\mathbf{1 4 / 2 0}$ \\
AtT18 & $0 / 20$ & $0 / 20$ & $\mathbf{1 3 / 2 0}$ & $0 / 20$ & $\mathbf{1 8 / 2 0}$ \\
AaGL3 & $\mathbf{2 5 / 2 8}$ & $\mathbf{1 6 / 2 8}$ & $\mathbf{2 4 / 2 8}$ & $0 / 28$ & $\mathbf{1 3 / 2 8}$ \\
AaEGL3 & $\mathbf{1 0 / 1 3}$ & $\mathbf{7 / 1 3}$ & $\mathbf{1 0 / 1 3}$ & $0 / 13$ & $\mathbf{( 6 / 1 3 ) ^ { \mathbf { a } }}$ \\
AaMYC1 & $0 / 10$ & $\mathbf{3 / 1 0}$ & $0 / 10$ & $0 / 10$ & $\mathbf{6 / 1 0}$ \\
AaTT8 & $0 / 20$ & $0 / 20$ & $\mathbf{1 2 / 2 0}$ & $0 / 20$ & $\mathbf{1 8 / 2 0}$ \\
GhDEL61 & $0 / 20$ & $\mathbf{1 2 / 2 0}$ & $\mathbf{9 / 2 0}$ & $0 / 20$ & $\mathbf{1 7 / 2 0}$ \\
GhDEL65 & $0 / 20$ & $\mathbf{1 3 / 2 0}$ & $\mathbf{1 0 / 2 0}$ & $0 / 20$ & $\mathbf{1 8 / 2 0}$ \\
PhAN1 & $0 / 20$ & $0 / 20$ & $\mathbf{1 1 / 2 0}$ & $0 / 20$ & $\mathbf{1 0 / 2 0}$ \\
PhJAF13 & $0 / 19$ & $\mathbf{1 0 / 1 9}$ & $0 / 19$ & $0 / 19$ & $\mathbf{1 8 / 1 9}$ \\
ZmR(LC) & $\mathbf{2 0 / 2 4}$ & $\mathbf{1 3 / 2 4}$ & $\mathbf{2 0 / 2 4}$ & $0 / 24$ & $\mathbf{( 1 0 / 2 4 ) ^ { \mathbf { a } }}$ \\
ZmR(S) & $\mathbf{2 0 / 2 2}$ & $\mathbf{1 5 / 2 2}$ & $\mathbf{2 0 / 2 2}$ & $0 / 22$ & $\mathbf{( 4 / 2 2 ) ^ { \mathbf { a } }}$ \\
ZmB & $0 / 10$ & $0 / 10$ & $0 / 10$ & $0 / 10$ & $0 / 10$ \\
\hline PaAtily & & & & &
\end{tabular}

apartially rescued. Bold values indicate rescue.

set of experiments in which the bHLH were expressed under the TT8 promoter in $t$ t 8 mutants and used these results for the interpretation (Table 3 ).

Consistent with the genetic results, AtGL3 rescued trichome and root hair formation and anthocyanidin production but not seed color or mucilage release (Supplementary Figures S3-S5 and Tables 2, 3). AtEGL3 rescued all four traits in which it is also genetically relevant and in addition shows a weak rescue of seed coat color. Consistent with its genetic function, TT8 expression led to a rescue of seed color and mucilage release (Supplementary Figures S5, S6). Thus, the differential function of the analyzed

TABLE 3 | T1 plants or T2 lines transformed by pAMPAT-proTT8:bHLH showing phenotypic rescue (rescued lines/total number of lines) in tt8 single mutant.

\begin{tabular}{lcc}
\hline Gene & Trichome & Seed coat color \\
\hline AtGL3 & $0 / 20$ & $0 / 20$ \\
AtEGL3 & $0 / 20$ & $\mathbf{( 5 / 2 0 ) ^ { \mathbf { b } }}$ \\
AtMYC1 & $0 / 20$ & $0 / 20$ \\
AtTT8 & $0 / 20$ & $\mathbf{1 8 / 2 0}$ \\
AaGL3 & $0 / 20$ & $0 / 20$ \\
AaEGL3 & $0 / 20$ & $0 / 20$ \\
AaMYC1 & $0 / 20$ & $0 / 20$ \\
AaTT8 & $0 / 20$ & $\mathbf{1 5 / 2 0}$ \\
GhDEL61 & $0 / 20$ & $0 / 20$ \\
GhDEL65 & $0 / 20$ & $0 / 20$ \\
PhAN1 & $0 / 20$ & $\mathbf{1 2 / 2 0}$ \\
PhJAF13 & $0 / 20$ & $0 / 20$ \\
ZmR(LC) & $0 / 20$ & $\mathbf{2 0 / 2 0}$ \\
ZmR(S) & $0 / 20$ & $\mathbf{1 9 / 2 0}$ \\
ZmB & $0 / 20$ & $0 / 20$ \\
\hline BEEd Can & &
\end{tabular}

${ }^{b}$ Seed coat is light brown. Bold values indicate rescue.
bHLH proteins can be explained at the protein level. The MYC1 protein, could rescue root hair and seed mucilage release.

To analyze the function of putative orthologous bHLH proteins we focused on another Brassicaceae species, A. alpina. Here, orthologous genes can be identified by sequence similarity and the chromosomal context of the respective genes by studying synteny. We found similar rescue properties for most bHLH proteins with one notable exception (Tables 2, 3). In contrast to AtGL3, the AaGL3 protein was unable to rescue the seed mucilage phenotype. This indicates that the function of GL3 has diverged between the two species at the protein level.

In cotton, we chose to analyze GhDEL65 and GhDEL61 that are linked to fiber formation and that can functionally replace AtGL3 and AtEGL3 in Arabidopsis when expressed under the Arabidopsis GL3 upstream sequences (Wang et al., 2013; Shangguan et al., 2016). We found rescue of the root hair, anthocyanidin and mucilage phenotype (Tables 2, 3). It is difficult to explain why trichome rescue was observed when the two cotton genes were expressed under the control of the AtGL3 upstream sequences but not under the control of the $35 \mathrm{~S}$ promoter in our studies. As the AtGL3 gene requires regulatory intron sequences for the correct expression (Friede et al., 2017), it is possible that the different results can be explained by unknown differences in the temporal, spatial or levels of expression. However, our data clearly show that the two bHLH proteins from cotton can regulate root hair, anthocyanidin and mucilage release. Hence, by their protein function they are most similar to Arabidopsis EGL3 and clearly distinct from TT8.

We analyzed two bHLH genes from Petunia, PhJAF13 and PhAN1. Mutations in PhAN1 lead to defects in anthocyanidin production, seed color and seed coat cell differentiation (Spelt et al., 2000, 2002). PhJAF13 was shown to activate anthocyanidin production upon overexpression (Quattrocchio et al., 1998). Our rescue experiments revealed different properties (Tables 2, 3). While PhAN1 expression in the gl3 egl3 tt8 mutant rescues the anthocyanidin, seed color and mucilage phenotypes, PhJAF13 overexpression resulted in a rescue of the root hair and seed coat mucilage phenotypes. Thus, the rescue ability of PhAN1 corresponds well with the mutant phenotype of the respective Petunia mutant. By contrast, PhJAF13 appears to have also the property to promote root hair formation (Supplementary Figure S3).

From Maize we selected three bHLH genes for our analysis, $Z m R(L c), Z m R(S)$, and $Z m B$. Mutations in all three genes result in severe anthocyanidin phenotypes (Dooner, 1979; Ludwig et al., 1989; Patterson et al., 1991; Stadler and Neuffer, 1953). Overexpression of both $Z m R$ genes was sufficient to rescue all five traits whereas $Z m B$ exhibited no rescue of any trait (Tables 2, 3).

\section{Rescue of the Arabidopsis Mutants by R2R3MYB Homologs}

We focused our analysis of the rescue ability of R2R3MYB proteins on the rescue of an anthocyanidin mutant as an example for the most ancient trait and trichome mutants as an example for a more recently evolved trait (Table 4). For the analysis of the anthocyanidin phenotype we used the 
TABLE 4 | T1 or T2 lines transformed by pAMPAT-35S-R2R3MYB showing phenotypic rescue (rescued lines/total number of lines) in g/1 single mutant or pap1pap2 mutant.

\begin{tabular}{|c|c|c|}
\hline Gene & Trichome (g/1) & Anthocyanin (pap1 pap2) \\
\hline AtGL1 & $17 / 18$ & $0 / 16$ \\
\hline AtWER & $0 / 16$ & $0 / 16$ \\
\hline AtPAP1 & $0 / 16$ & $18 / 18$ \\
\hline AtPAP2 & $0 / 16$ & $13 / 18$ \\
\hline AtTT2 & $(9 / 16)^{c}$ & $0 / 16$ \\
\hline AtMYB61 & 0/16 & $0 / 16$ \\
\hline AaGL1 & $14 / 16$ & $0 / 16$ \\
\hline AaWER & $0 / 16$ & $0 / 16$ \\
\hline AaPAPL & $0 / 16$ & $17 / 18$ \\
\hline AaMYB23 & $0 / 16$ & $0 / 16$ \\
\hline GhMYB2 & $14 / 16$ & $0 / 15$ \\
\hline GhMYB3 & $15 / 16$ & $0 / 15$ \\
\hline GhMYB25 & 0/16 & $0 / 15$ \\
\hline GhRLC1 & $0 / 16$ & $6 / 12$ \\
\hline PhAN2 & $0 / 16$ & $10 / 16$ \\
\hline PhAN4 & $0 / 16$ & $13 / 16$ \\
\hline $\mathrm{PhPH} 4$ & $0 / 16$ & $7 / 14$ \\
\hline$Z m C 1$ & 0/16 & $8 / 12$ \\
\hline$Z m P L$ & $(6 / 16)^{c}$ & $7 / 12$ \\
\hline$Z m P 1$ & $0 / 16$ & $9 / 15$ \\
\hline
\end{tabular}

${ }^{c}$ Trichomes on leaf margin. Bold values indicate rescue.

Arabidopsis pap1 pap2 double mutant. AtPAP1 and AtPAP2 are close homologs and both involved in the regulation of anthocyanidin (Teng et al., 2005; Gonzalez et al., 2008). The trichome phenotype was assessed in Arabidopsis gll mutants. The R2R3MYB proteins were expressed under the control of the $35 \mathrm{~S}$ promoter. As expected both, AtPAP1 and AtPAP2, rescued the double mutant efficiently. However, none of the other Arabidopsis R2R3MYB proteins including AtGL1, AtWER, AtTT2, and AtMYB61 were able to rescue the anthocyanidin phenotype. The gll trichome phenotype was rescued only by GL1 but not by any of the other proteins (Supplementary Figure S7). This suggest that also the R2R3MYB protein have evolved trait specific properties. A similar result was obtained for the Arabis R2R3MYB proteins. Only the PAPlike protein AaPAPL revealed rescue of the anthocyanidin phenotype and only AaGL1 the gll phenotype. In cotton we chose four R2R3MYB proteins. GhMYB2 is expressed in fibers and can rescue the Arabidopsis trichome phenotype (Wang et al., 2004). GhMYB25 is expressed in the seed coat and mutations in the gene lead to slower fiber growth (Machado et al., 2009). GhRLC1 was shown to be involved in the regulation of anthocyanidin in cotton (Gao et al., 2013). In addition we added GhMYB3 which we found as a close homolog of GhMYB2. Consistent with the mutant phenotype, GhRLC1 rescued the anthocyanidin phenotype (Table 4). GhMYB2 and GhMYB3 rescued the gl1 trichome phenotype, GhMYB25 exhibited no rescue. In Petunia we analyzed three genes. PhAN2 and PhAN4 are important for anthocyanidin production (Quattrocchio et al., 1993, 2006b; Spelt et al., 2002). PhPH4 regulates the acidification of the vacuole in petals (Quattrocchio et al., 2006b). All three R2R3MYB proteins rescued the anthocyanidin phenotype but not the trichome phenotype. Finally, we studied the $\mathrm{ZmC1}$ gene from maize that was described to act as a regulator of anthocyanidin production (Sainz et al., 1997; Grotewold et al., 2000; Hernandez et al., 2004). Consistent with this function in maize we found that $Z m C 1$ rescued the pap1 pap2 mutant but not the gl1 mutant.

\section{Inter-Species MBW Pairwise Interaction}

It is generally thought that the specificity of MBW function is due to the formation of specific combinations of particular components. In this light, the differential rescue behavior of MBW proteins from different species might be explained by differences in the protein-protein interactions with the proteins in A. thaliana. Interactions of MBW proteins in each species were already extensively studied (Supplementary Table S3). To analyze the interactions of MBW proteins from other species with the Arabidopsis proteins we performed LUMIER assays [LUminescence-based Mammalian IntERactome (Blasche and Koegl, 2013)]. In LUMIER assays proteins of interest are expressed in human HEK293TM cells with one protein fused to the $S$. aureus protein A (ProtA) and the other with a $R$. reniformis luciferase (Rluc) followed by pulldown experiments and a quantification of the precipitated luciferase activity (Supplementary Figure S8 and Table 5).

In a first step we explored the binding behavior of TTG1 homologs to Arabidopsis bHLH proteins (Table 5). AaTTG1, GhTTG1, PhAN11, and ZmPAC1 exhibited interactions with all four Arabidopsis bHLH proteins. GhTTG3 interacted with AtGL3, AtEGL3, and AtMYC1 but not with AtTT8. GhTTG2, GhTTG4, and ZmMP1 proteins did not interact with any of the bHLH proteins. This correlates well with our finding that the latter four TTG1 homologs did not rescue ttg1 mutants in Arabidopsis.

In a next step we tested the binding of homologous bHLH proteins to Arabidopsis TTG1 and MYB proteins (Table 5). All bHLH proteins tested showed an interaction with the Arabidopsis AtTTG1 protein. Binding of bHLH proteins from other species with the Arabidopsis R2R3MYB proteins varied. Most bHLH proteins showed interaction with GL1, WER, PAP1, PAP2, and TT2 but not with MYB61. Arabis EGL3 exhibited a differential binding behavior such that it interacts with GL1, WER, PAP1, and TT2 but not PAP2. We found no interactions of $\mathrm{ZmB}$ with any $\mathrm{R} 2 \mathrm{R} 3 \mathrm{MYBs}$. This is consistent with the fact that $\mathrm{ZmB}$ possesses no rescue ability.

Finally, we studied the binding behavior of R2R3MYB from other species with Arabidopsis MBW proteins (Table 5). As expected, we generally found no binding to AtTTG1. Only ZmPL exhibited weak binding to AtTTG1. Except for GhMYB25 all R2R3MYB proteins interacted with all four bHLH proteins from Arabidopsis.

Taken together the analysis of the interactions of MBW proteins from other species with the Arabidopsis proteins can explain those cases in which we found no rescue of Arabidopsis mutants as the respective proteins do not interact with the 
TABLE 5 | Analysis of inter-species MBW pairwise interaction by LUMIER pulldown assays.

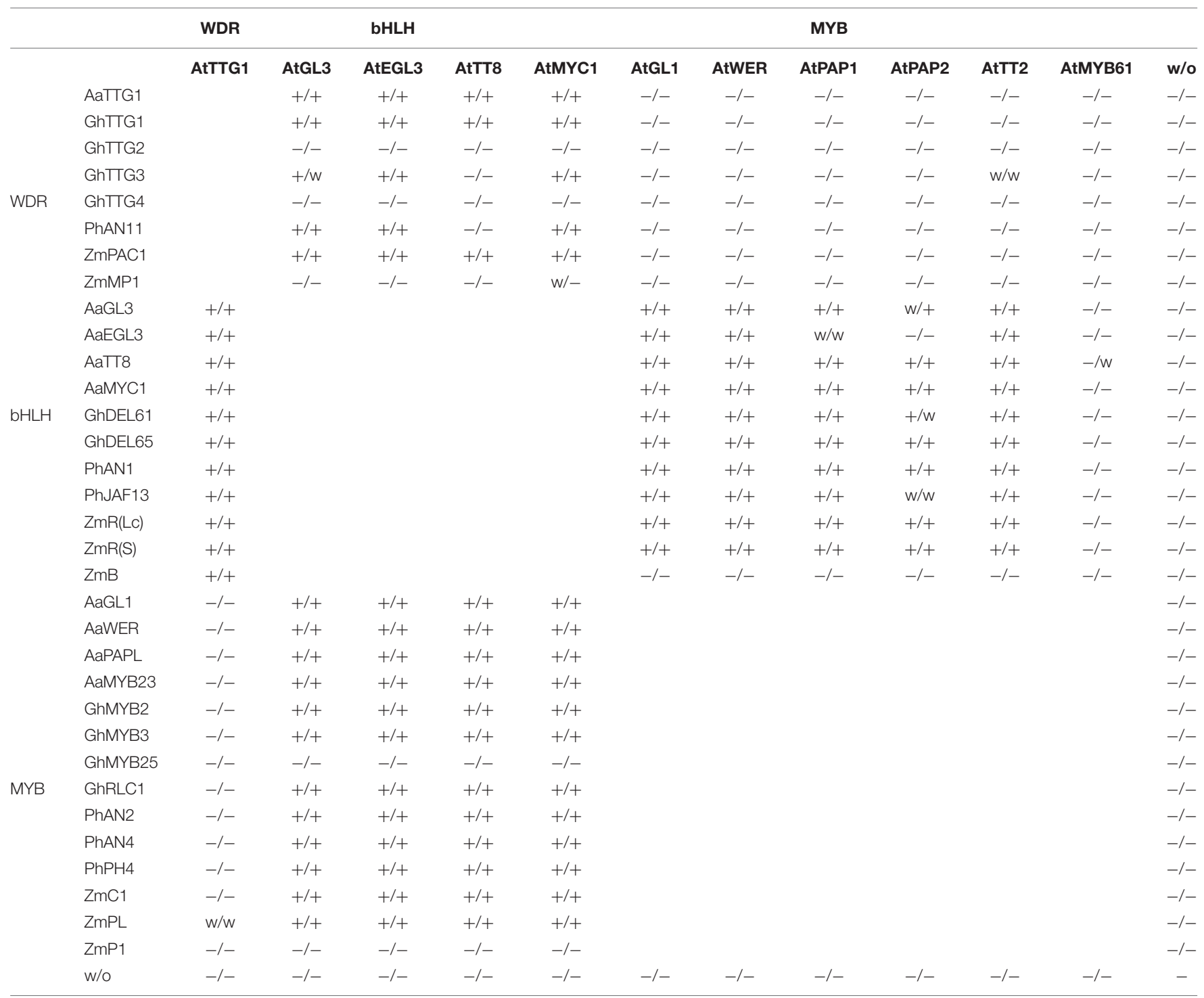

Arabidopsis thaliana [At], Arabis alpine [Aa], Gossypium hirsutum [Gh], Petunia hybrid [Ph] and Zea mays [Zm]. All proteins are single-expressed in human cells (HEK293TN) and immunoprecipitated with IgG Dynabeads (For full set of data please refer to Supplementary Tables S4, S5). w/o: Empty vector without CDS fusion. +: positive interaction (luciferase activity $\geq 2.5 \%$ ). w: weak interaction (luciferase activity $=1.5 \% \sim 2.5 \%$ ). - : no interaction (luciferase activity $<1.5 \%$ ).

relevant partners. However, the binding patterns do not explain any differential rescue of traits.

\section{DISCUSSION}

\section{Rescue With Arabidopsis MBW Proteins Reveals Trait-Specific Protein Properties}

In Arabidopsis, the MBW complex regulates five different traits by involving different bHLH and R2R3MYBs in a trait-specific manner. This raises the question, whether trait specificity is due to transcriptional regulation or different properties of bHLH and R2R3MYB proteins. There are examples available suggesting that both aspects are relevant (Lai et al., 2013; Lloyd et al., 2017). To address this question, we expressed the MBW proteins under the
35S promoter in appropriate A. thaliana mutants and analyzed, which traits were rescued in the T1 generation. This enabled us to judge the ability of MBW proteins to function in the regulation of the different traits. Rescue experiments with $A$. thaliana bHLH proteins GL3, EGL3, and TT8 revealed that the rescue ability of the different traits matches their genetic requirements (Supplementary Table S1) (Zhang et al., 2003). This suggests that these bHLH proteins possess properties mediating trait specific regulation events. By contrast, the bHLH protein MYC1 is important for trichome development but cannot rescue the trichome phenotype of the $g l 3$ egl 3 tg 8 mutant. This is consistent with previous data (Zhao et al., 2012) and can be explained by a different cellular localization and molecular function (Pesch et al., 2013). Similarly, we found some trait specificity for the R2R3MYB proteins. However, we found no rescue of gl1 mutants 
with overexpressed WER. This is in conflict with previous findings where WER expression under the GL1 promoter showed rescue (Lee and Schiefelbein, 2001). This may be considered to show the limitations of the overexpression approach taken in this study. It may also reflect that an overexpression approach is more stringent such that only GL1 can rescue the gll mutant but not WER.

\section{Evolutionary Comparison of MBW Functions}

Because a rescue approach considering all five traits does not allow a systematic analysis of evolutionary conserved MBW proteins, we selected genes and species for which genetic or molecular data are available suggesting a functional relevance in one or several of the pathways under consideration. As a consequence, some of our experiments are repetitions and can serve as references and controls for comparison with previous results. Most WDR homologs studied here were able to complement $\operatorname{tg} 1 \mathrm{~A}$. thaliana mutants. Only the two cotton proteins GhTTG2 and GhTTG4 and the maize MP1 protein did not show any rescue. This observation correlates with the phylogenetic classification that places MP1 and PAC1 in different clades (Carey et al., 2004). All WDR proteins including AtTTG1, AaTTG1, GhTTG1, GhTTG2, GhTTG2, and GhTTG4 are most similar to AtAN11, which belongs to MP1 clade (Carey et al., 2004; Humphries et al., 2005). The rescue ability of bHLH protein from other species correlates reasonably well with the function of those for which mutant data are available. This is true for the A. alpina proteins TT8, EGL3, petunia AN1 and maize R(LC) and $\mathrm{R}(\mathrm{S})$. A notable exception is the maize $\mathrm{ZmB}$ protein as the corresponding maize mutants show an anthocyanidin phenotype. As the $\mathrm{ZmB}$ protein does not interact with any of the A. thaliana $\mathrm{R} 2 \mathrm{R} 3 \mathrm{MYB}$ proteins, it is conceivable that $\mathrm{ZmB}$ cannot rescue because of this lack of interactions. We found a similar situation for the R2R3MYB proteins. Here all R2R3MYBs for which a function in the regulation of anthocyanidin was demonstrated by mutant phenotypes in the respective species specifically rescued the anthocyanidin phenotype but not the trichome phenotype. This includes the cotton proteins GhRCL1 (Gao et al., 2013), the petunia proteins PhAN2 and PhAN4 (Quattrocchio et al., 1993, 2006b; Spelt et al., 2002) and the maize protein ZmC1 (Sainz et al., 1997; Grotewold et al., 2000; Hernandez et al., 2004).

Previous experiments aiming to understand whether changes in the temporal-spatial regulation or changes in protein functions are relevant to adopt trait specific functions revealed examples for an importance of gene regulation. GL1 and WER can equally

\section{REFERENCES}

Baudry, A., Caboche, M., and Lepiniec, L. (2006). TT8 controls its own expression in a feedback regulation involving TTG1 and homologous MYB and bHLH factors, allowing a strong and cell-specific accumulation of flavonoids in Arabidopsis thaliana. Plant J. 46, 768-779. doi: 10.1111/j.1365-313X.2006. 02733.x

Baudry, A., Heim, M. A., Dubreucq, B., Caboche, M., Weisshaar, B., and Lepiniec, L. (2004). TT2, TT8, and TTG1 synergistically specify the expression well rescue the wer root hair phenotype when expressed under the WER promoter (Lee and Schiefelbein, 2001). Similarly, the gl1 mutant phenotype can be rescued by the MYB82 protein when expressed under the GL1 promoter (Liang et al., 2014). Taken together, our data suggest that also changes in protein functions of MBW proteins are important. The specificity for the regulation of the five the different TTG1-dependent traits can be explained to a large degree by the protein behavior of the bHLH and R2R3MYB proteins. It will be interesting to see in future experiments to understand which biochemical properties of the bHLH and R2R3MYB proteins might be relevant for trait specific regulation.

\section{DATA AVAILABILITY}

All datasets generated for this study are included in the manuscript and/or the Supplementary Files.

\section{AUTHOR CONTRIBUTIONS}

$\mathrm{BZ}$ performed the experiments. $\mathrm{BZ}$ and $\mathrm{MH}$ designed the experiments and wrote the manuscript.

\section{FUNDING}

This work was funded by the DFG priority program SFB 680 .

\section{ACKNOWLEDGMENTS}

We thank Anna Deneer for critical reading of the manuscript. We thank Prof. Dr. Ronald Koes for providing petunia cDNA constructs of AN11, AN1, JAF13, AN2, AN4, and PH4. We are grateful for Dr. Jing Qu providing us Gossypium hirsutum, cultivar TM-1 seed (Chinese Academy of Sciences, China). We thank Sabine Lohmer for technical help and Divikry Chopra, Andrea Schrader, Heike Wolff, and Johannes Span for providing Arabis alpina clones.

\section{SUPPLEMENTARY MATERIAL}

The Supplementary Material for this article can be found online at: https://www.frontiersin.org/articles/10.3389/fpls.2019.00375/ full\#supplementary-material

of BANYULS and proanthocyanidin biosynthesis in Arabidopsis thaliana. Plant J. 39, 366-380. doi: 10.1111/j.1365-313X.2004.02138.x

Bernhardt, C., Lee, M. M., Gonzalez, A., Zhang, F., Lloyd, A., and Schiefelbein, J. (2003). The bHLH genes Glabra3 (GL3) and Enhancer of Glabra3 (EGL3) specify epidermal cell fate in the Arabidopsis root. Development 130, 6431-6439. doi: 10.1242/dev.00880

Blasche, S., and Koegl, M. (2013). Analysis of protein-protein interactions using LUMIER assays. Methods Mol. Biol. 1064, 17-27. doi: 10.1007/978-1-62703601-6_2 
Borevitz, J. O., Xia, Y., Blount, J., Dixon, R. A., and Lamb, C. (2000). Activation tagging identifies a conserved MYB regulator of phenylpropanoid biosynthesis. Plant Cell 12, 2383-2393. doi: 10.1105/tpc.12.12.2383

Bruex, A., Kainkaryam, R. M., Wieckowski, Y., Kang, Y. H., Bernhardt, C., Xia, Y., et al. (2012). A gene regulatory network for root epidermis cell differentiation in Arabidopsis. PLoS Genet. 8:e1002446. doi: 10.1371/journal.pgen.1002446

Carey, C. C., Strahle, J. T., Selinger, D. A., and Chandler, V. L. (2004). Mutations in the pale aleurone color 1 regulatory gene of the Zea mays anthocyanin pathway have distinct phenotypes relative to the functionally similar Transparent Testa Glabral gene in Arabidopsis thaliana. Plant Cell 16, 450-464. doi: 10.1105/tpc. 018796

Chopra, D., Wolff, H., Span, J., Schellmann, S., Coupland, G., Albani, M. C., et al. (2014). Analysis of TTG1 function in Arabis alpina. BMC Plant Biol. 14:16. doi: 10.1186/1471-2229-14-16

Clough, S. J., and Bent, A. F. (1998). Floral dip: a simplified method for Agrobacterium-mediated transformation of Arabidopsis thaliana. Plant J. 16, 735-743. doi: 10.1046/j.1365-313x.1998.00343.x

Dooner, H. K. (1979). Identification of an $<\mathrm{em}>\mathrm{r}</ \mathrm{em}>$-locus region that controls the tissue specificity of anthocyanin formation in maize. Genetics 93 , $703-710$.

Dressel, A., and Hemleben, V. (2009). Transparent Testa Glabra 1 (TTG1) and TTG1-like genes in Matthiola incana R. Br. and related Brassicaceae and mutation in the WD-40 motif. Plant Biol. 11, 204-212. doi: 10.1111/j.14388677.2008.00099.x

Feller, A., Hernandez, J. M., and Grotewold, E. (2006). An ACT-like domain participates in the dimerization of several plant basic-helix-loop-helix transcription factors. J. Biol. Chem. 281, 28964-28974. doi: 10.1074/jbc. M603262200

Feyissa, D. N., Løvdal, T., Olsen, K. M., Slimestad, R., and Lillo, C. (2009). The endogenous GL3, but not EGL3, gene is necessary for anthocyanin accumulation as induced by nitrogen depletion in Arabidopsis rosette stage leaves. Planta 230:747. doi: 10.1007/s00425-009-0978-3

Friede, A., Zhang, B., Herberth, S., Pesch, M., Schrader, A., and Hulskamp, M. (2017). The second intron is essential for the transcriptional control of the Arabidopsis thaliana Glabra3 gene in leaves. Front Plant Sci 8:1382. doi: 10.3389/ fpls.2017.01382

Gao, Z., Liu, C., Zhang, Y., Li, Y., Yi, K., Zhao, X., et al. (2013). The promoter structure differentiation of a MYB transcription factor RLC1 causes red leaf coloration in empire red leaf cotton under light. PLoS One 8:e77891. doi: 10. 1371/journal.pone.0077891

Gonzalez, A., Mendenhall, J., Huo, Y., and Lloyd, A. (2009). TTG1 complex MYBs, MYB5 and TT2, control outer seed coat differentiation. Dev. Biol. 325, 412-421. doi: 10.1016/j.ydbio.2008.10.005

Gonzalez, A., Zhao, M., Leavitt, J. M., and Lloyd, A. M. (2008). Regulation of the anthocyanin biosynthetic pathway by the TTG1/bHLH/Myb transcriptional complex in Arabidopsis seedlings. Plant J. 53, 814-827. doi: 10.1111/j.1365313X.2007.03373.x

Grotewold, E., Sainz, M. B., Tagliani, L., Hernandez, J. M., Bowen, B., and Chandler, V. L. (2000). Identification of the residues in the Myb domain of maize $\mathrm{C} 1$ that specify the interaction with the bHLH cofactor R. Proc. Natl. Acad. Sci. U.S.A. 97, 13579-13584. doi: 10.1073/pnas.25037 9897

Heppel, S. C., Jaffe, F. W., Takos, A. M., Schellmann, S., Rausch, T., Walker, A. R., et al. (2013). Identification of key amino acids for the evolution of promoter target specificity of anthocyanin and proanthocyanidin regulating MYB factors. Plant Mol. Biol. 82, 457-471. doi: 10.1007/s11103-013-0074-8

Herman, P. L., and Marks, M. D. (1989). Trichome development in Arabidopsis thaliana. II. Isolation and complementation of the GLABROUS1 Gene. Plant Cell 1, 1051-1055. doi: 10.1105/tpc.1.11.1051

Hernandez, J. M., Heine, G. F., Irani, N. G., Feller, A., Kim, M.-G., Matulnik, T., et al. (2004). Different mechanisms participate in the R-dependent activity of the R2R3 MYB transcription factor C1. J. Biol. Chem. 279, 48205-48213. doi: 10.1074/jbc.M407845200

Hülskamp, M., Miséra, S., and Jürgens, G. (1994). Genetic dissection of trichome cell development in Arabidopsis. Cell 76, 555-566. doi: 10.1016/0092-8674(94) 90118-X

Humphries, J. A., Walker, A. R., Timmis, J. N., and Orford, S. J. (2005). Two WDrepeat genes from cotton are functional homologues of the Arabidopsis thaliana
TRANSPARENT TESTA GLABRA1 (TTG1) gene. Plant Mol. Biol. 57, 67-81. doi: 10.1007/s11103-004-6768-1

Jakoby, M. J., Falkenhan, D., Mader, M. T., Brininstool, G., Wischnitzki, E., Platz, N., et al. (2008). Transcriptional profiling of mature Arabidopsis trichomes reveals that $<\mathrm{em}>$ NOECK $</ \mathrm{em}>$ encodes the MIXTA-like transcriptional regulator MYB106. Plant Physiol. 148, 1583-1602. doi: 10.1104/ pp.108.126979

Jones, D. T., Taylor, W. R., and Thornton, J. M. (1992). The rapid generation of mutation data matrices from protein sequences. Bioinformatics 8, 275-282. doi: 10.1093/bioinformatics/8.3.275

Kirik, V., Lee, M. M., Wester, K., Herrmann, U., Zheng, Z., Oppenheimer, D., et al. (2005). Functional diversification of MYB23 and GL1 genes in trichome morphogenesis and initiation. Development 132, 1477-1485. doi: 10.1242/dev. 01708

Koornneef, M. (1981). The complex syndrome of ttg mutants. Arabidopsis Inf. Serv. $18,45-51$.

Kuromori, T., Hirayama, T., Kiyosue, Y., Takabe, H., Mizukado, S., Sakurai, T., et al. (2004). A collection of 11800 single-copy Ds transposon insertion lines in Arabidopsis. Plant J. 37, 897-905. doi: 10.1111/j.1365.313X.2004.02009.x

Lai, Y., Li, H., and Yamagishi, M. (2013). A review of target gene specificity of flavonoid R2R3-MYB transcription factors and a discussion of factors contributing to the target gene selectivity. Front. Biol. 8:577-598. doi: 10.1007/ s11515-013-1281-z

Lee, M. M., and Schiefelbein, J. (1999). WEREWOLF, a MYB-related protein in < em $>$ Arabidopsis $</$ em $>$, Is a position-dependent regulator of epidermal cell patterning. Cell 99, 473-483. doi: 10.1016/S0092-8674(00)81536-6

Lee, M. M., and Schiefelbein, J. (2001). Developmentally distinct MYB genes encode functionally equivalent proteins in Arabidopsis. Development 128, 15391546.

Liang, G., He, H., Li, Y., Ai, Q., and Yu, D. (2014). MYB82 functions in regulation of trichome development in Arabidopsis. J. Exp. Bot. 65, 3215-3223. doi: 10. 1093/jxb/eru179

Lloyd, A., Brockman, A., Aguirre, L., Campbell, A., Bean, A., Cantero, A., et al. (2017). Advances in the MYB-bHLH-WD repeat (MBW) pigment regulatory model: addition of a WRKY factor and co-option of an anthocyanin MYB for betalain regulation. Plant Cell Physiol. 58, 1431-1441. doi: 10.1093/pcp/pcx075

Ludwig, S. R., Habera, L. F., Dellaporta, S. L., and Wessler, S. R. (1989). Lc, a member of the maize R gene family responsible for tissue-specific anthocyanin production, encodes a protein similar to transcriptional activators and contains the myc-homology region. Proc. Natl. Acad. Sci. U.S.A. 86, 7092-7096. doi: 10.1073/pnas.86.18.7092

Machado, A., Wu, Y., Yang, Y., Llewellyn, D. J., and Dennis, E. S. (2009). The MYB transcription factor GhMYB25 regulates early fibre and trichome development. Plant J. 59, 52-62. doi: 10.1111/j.1365-313X.2009.03847.x

Marks, M. D., and Feldmann, K. A. (1989). Trichome Development in Arabidopsis thaliana. I. T-DNA Tagging of the GLABROUS1 Gene. Plant Cell 1, 1043-1050. doi: 10.1105/tpc.1.11.1043

Nemesio-Gorriz, M., Blair, P. B., Dalman, K., Hammerbacher, A., Arnerup, J., Stenlid, J., et al. (2017). Identification of norway spruce MYB-bHLH-WDR transcription factor complex members linked to regulation of the flavonoid pathway. Front. Plant Sci. 8:305. doi: 10.3389/fpls.2017.00305

Nesi, N., Debeaujon, I., Jond, C., Pelletier, G., Caboche, M., and Lepiniec, L. (2000). The TT8 gene encodes a basic helix-loop-helix domain protein required for expression of DFR and BAN genes in Arabidopsis Siliques. Plant Cell 12, 1863-1878. doi: $10.1105 /$ tpc. 12.10 .1863

Oppenheimer, D. G., Herman, P. L., Sivakumaran, S., Esch, J., and Marks, M. D. (1991). A myb gene required for leaf trichome differentiation in Arabidopsis is expressed in stipules. Cell 67, 483-493. doi: 10.1016/0092-8674(91)90523-2

Patterson, G. I., Harris, L. J., Walbot, V., and Chandler, V. L. (1991). Genetic analysis of B-Peru, a regulatory gene in maize. Genetics 127, 205-220.

Payne, C. T., Zhang, F., and Lloyd, A. M. (2000). GL3 encodes a bHLH protein that regulates trichome development in Arabidopsis through interaction with GL1 and TTG1. Genetics 156, 1349-1362.

Penfield, S., Meissner, R. C., Shoue, D. A., Carpita, N. C., and Bevan, M. W. (2001). MYB61 is required for mucilage deposition and extrusion in the Arabidopsis seed coat. Plant Cell 13, 2777-2791. doi: 10.1105/tpc.13.12.2777

Pesch, M., Schultheiss, I., Klopffleisch, K., Uhrig, J. F., Koegl, M., Clemen, C. S., et al. (2015). TRANSPARENT TESTA GLABRA1 and GLABRA1 compete for 
binding to GLABRA3 in Arabidopsis. Plant Physiol. 168, 584-597. doi: 10.1104/ pp. 15.00328

Pesch, M., Schultheiß, I., Digiuni, S., Uhrig, J. F., and Hülskamp, M. (2013). Mutual control of intracellular localisation of the patterning proteins AtMYC1, GL1 and TRY/CPC in Arabidopsis. Development 140, 3456-3467. doi: 10.1242/dev. 094698

Quattrocchio, F., Baudry, A., Lepiniec, L., and Grotewold, E. (2006a). "The regulation of flavonoid biosynthesis," in The Science of Flavonoids, ed. E. Grotewold (New York, NY: Springer), 97-122.

Quattrocchio, F., Verweij, W., Kroon, A., Spelt, C., Mol, J., and Koes, R. (2006b). PH4 of petunia is an R2R3 MYB protein that activates vacuolar acidification through interactions with basic-helix-loop-helix transcription factors of the anthocyanin pathway. Plant Cell 18, 1274-1291.

Quattrocchio, F., Wing, J. F., Leppen, H., Mol, J., and Koes, R. E. (1993). Regulatory genes controlling anthocyanin pigmentation are functionally conserved among plant species and have distinct sets of target genes. Plant Cell 5, 1497-1512. doi: $10.1105 /$ tpc.5.11.1497

Quattrocchio, F., Wing, J. F., van der Woude, K., Mol, J. N., and Koes, R. (1998). Analysis of bHLH and MYB domain proteins: species-specific regulatory differences are caused by divergent evolution of target anthocyanin genes. Plant J. 13, 475-488. doi: 10.1046/j.1365-313X.1998.00046.x

Ramsay, N. A., and Glover, B. J. (2005). MYB-bHLH-WD40 protein complex and the evolution of cellular diversity. Trends Plant Sci. 10, 63-70. doi: 10.1016/j. tplants.2004.12.011

Sainz, M. B., Grotewold, E., and Chandler, V. L. (1997). Evidence for direct activation of an anthocyanin promoter by the maize $\mathrm{C} 1$ protein and comparison of DNA binding by related Myb domain proteins. Plant Cell 9, 611-625. doi: $10.1105 /$ tpc.9.4.611

Serna, L., and Martin, C. (2006). Trichomes: different regulatory networks lead to convergent structures. Trends Plant Sci. 11, 274-280. doi: 10.1016/j.tplants. 2006.04.008

Shangguan, X.-X., Yang, C.-Q., Zhang, X.-F., and Wang, L.-J. (2016). Functional characterization of a basic helix-loop-helix (bHLH) transcription factor GhDEL65 from cotton (Gossypium hirsutum). Physiol. Plant. 158, 200-212. doi: $10.1111 /$ ppl.12450

Spelt, C., Quattrocchio, F., Mol, J., and Koes, R. (2002). ANTHOCYANIN1 of petunia controls pigment synthesis, vacuolar $\mathrm{pH}$, and seed coat development by genetically distinct mechanisms. Plant Cell 14, 2121-2135. doi: 10.1105/tpc. 003772

Spelt, C., Quattrocchio, F., Mol, J. N. M., and Koes, R. (2000). anthocyanin1 of petunia encodes a basic helix-loop-helix protein that directly activates transcription of structural anthocyanin genes. Plant Cell 12, 1619-1631. doi: 10.1105/tpc.12.9.1619

Stadler, L. J., and Neuffer, M. G. (1953). Problems of gene structure. II. Separation of R Elements (S) and (P) by unequal crossing over. Science 117, 471-472.

Symonds, V. V., Hatlestad, G., and Lloyd, A. M. (2011). Natural allelic variation defines a role for ATMYC1: trichome cell fate determination. PLoS Genet. 7:e1002069. doi: 10.1371/journal.pgen.1002069
Tamura, K., Stecher, G., Peterson, D., Filipski, A., and Kumar, S. (2013). MEGA6: molecular evolutionary genetics analysis version 6.0. Mol. Biol. Evol. 30, 27252729. doi: 10.1093/molbev/mst197

Teng, S., Keurentjes, J., Bentsink, L., Koornneef, M., and Smeekens, S. (2005). Sucrose-specific induction of anthocyanin biosynthesis in Arabidopsis requires the MYB75/PAP1 gene. Plant Physiol. 139, 1840-1852. doi: 10.1104/pp.105. 066688

Walker, A. R., Davison, P. A., Bolognesi-Winfield, A. C., James, C. M., Srinivasan, N., Blundell, T. L., et al. (1999). The TRANSPARENT TESTA GLABRA1 locus, which regulates trichome differentiation and anthocyanin biosynthesis in Arabidopsis, encodes a WD40 repeat protein. Plant Cell 11, 1337-1349. doi: 10.1105/tpc.11.7.1337

Wang, G., Zhao, G.-H., Jia, Y.-H., and Du, X.-M. (2013). Identification and characterization of cotton genes involved in fuzz-fiber development. J. Integr. Plant Biol. 55, 619-630. doi: 10.1111/jipb.12072

Wang, S., Wang, J. W., Yu, N., Li, C. H., Luo, B., Gou, J. Y., et al. (2004). Control of plant trichome development by a cotton fiber MYB gene. Plant Cell 16, 2323-2334. doi: 10.1105/tpc.104.024844

Xu, W. J., Dubos, C., and Lepiniec, L. (2015). Transcriptional control of flavonoid biosynthesis by MYB-bHLH-WDR complexes. Trends Plant Sci. 20, 176-185. doi: 10.1016/j.tplants.2014.12.001

Zhang, B., and Schrader, A. (2017). TRANSPARENT TESTA GLABRA 1dependent regulation of flavonoid biosynthesis. Plants 6:E65. doi: 10.3390/ plants6040065

Zhang, F., Gonzalez, A., Zhao, M., Payne, C. T., and Lloyd, A. (2003). A network of redundant bHLH proteins functions in all TTG1-dependent pathways of Arabidopsis. Development 130, 4859-4869. doi: 10.1242/dev.00681

Zhao, H., Wang, X., Zhu, D., Cui, S., Li, X., Cao, Y., et al. (2012). A single amino acid substitution in IIIf subfamily of basic helix-loophelix transcription factor AtMYC1 leads to trichome and root hair patterning defects by abolishing its interaction with partner proteins in Arabidopsis. J. Biol. Chem. 287, 14109-14121. doi: 10.1074/jbc.M111.28 0735

Zimmermann, I. M., Heim, M. A., Weisshaar, B., and Uhrig, J. F. (2004). Comprehensive identification of Arabidopsis thaliana MYB transcription factors interacting with R/B-like BHLH proteins. Plant J. 40, 22-34. doi: 10. 1111/j.1365-313X.2004.02183.x

Conflict of Interest Statement: The authors declare that the research was conducted in the absence of any commercial or financial relationships that could be construed as a potential conflict of interest.

Copyright (๑) 2019 Zhang and Hülskamp. This is an open-access article distributed under the terms of the Creative Commons Attribution License (CC BY). The use, distribution or reproduction in other forums is permitted, provided the original author(s) and the copyright owner(s) are credited and that the original publication in this journal is cited, in accordance with accepted academic practice. No use, distribution or reproduction is permitted which does not comply with these terms. 